\title{
MODELING, SIMULATION AND NEURAL CONTROL OF WHEELED INVERTED PENDULUM
}

\author{
Muhammad Yasir Amir Khan ${ }^{1}$, Vali Uddin Abbas ${ }^{2}$ \\ 1 Department of Electrical Engineering Bahria University, Pakistan \\ 2 Department of Electrical Engineering, Hamdard University, Pakistan \\ Email:engr.yasir.niazi@gmail.com
}

\begin{abstract}
Wheeled inverted pendulum (WIP) is an underactuated and a highly unstable system. In this paper we have proposed modelling and control of WIP as hybrid automaton based on torque equations rather than Lagrangian method. The model was simulated using Simulink ${ }^{\oplus}$ using Direct Inverse Neural Control (DINC) to control the WIP system using the hybrid automaton based strategy so as to keep it at upright position. Simulation results reveal that control scheme is sufficiently able to control the system at desired position but with slight steady state error which was found to reduce with the increasing sampling rate.
\end{abstract}

Keywords: Quadrotor, Backpropagation, Perceptron.

\section{INTRODUCTION}

The type of robot investigated in this paper is a mobile robot with an inverted pendulum and a pair of wheels. Such a system is called wheeled inverted pendulum (WIP) system in literature. WIP robot is often referred to as Segbot, Segway or a Two-Wheeled Selfbalancing Vehicle. This design was selected in this research work because mechanism of this robot has a rather interesting motion for stabilizing robot's orientation, as it has a pair of wheels for moving on a plane surface, it therefore quite resembles the standing and walking posture of humans. In WIP, the two independent wheels are co-axial and placed on the left and right hand side of the robot. These wheels are used for controlling the position of the machine [1].

WIP is an under-actuated nonlinear system. By definition, an under-actuated system is a physical system which possesses lesser number of actuators than the degrees of freedom. In this robot there are two actuators in the form of wheels on either side of it. On the other hand the number of degrees of freedom is more than two, which includes pitch, yaw motion, and two dimensional planner motions. The WIP system may initially be mistaken to be similar to an inverted pendulum-cart system. It must be noted that the dynamics of both systems are quite different. In case of an inverted pendulum on a cart, the pendulum is cart mounted with tilt-angular motion with respect to cart and the actuator may be mounted on the cart or may not even be on board (i.e. belt driven system), whereas in a WIP system, the motors are mounted on the pendulum. Therefore reaction torque on motor is directly applied on the pendulum itself. Moreover in case of WIP both wheels can be driven independently resulting in yaw motion in addition to the translational motion [2]. 


\section{LITERATURE REVIEW}

In the past, research has been done with more focus on certain types of robots such as crawling robot, flying robot and others. Nevertheless, a good amount of research work has also been carried out on WIP type of robot, which has been discussed in this paper. The first WIP robot was constructed by Professor Kazuo Yamafuji of University of Electro-Communications in Tokyo, in 1986 [3]. The robot designed and fabricated under his supervision could "simulate" the working of an inverted pendulum but it had a rolling cart attached to a pair of wheels.

David P. Anderson developed nBOT. This WIP system used data obtained from the accelerometer and gyroscope. The data collected from the inertial measurement unit was filtered and combined to give a single measurement of the tilt angle [5]. Another interesting work developed by Dani Piponi [6] was Equibot. This robot used infrared (IR) to determine the distance from the ground and used this information to deduce its tilt angle.

Yet another interesting work is Segbot built in 2004 with an intention to build a self balancing robot based on working principles of Segway [7]. There are many other interesting attempts as well, in building WIP system such as Segway, Two-Wheel Vehicle B2, the Joe, CMU Ballbot, uBot etc. to name a few [4].

\section{PROBLEMS IN CONTROLLING WIP SYSTEM}

This WIP system is an example of a under actuated system where the plant has less control inputs compared to the generalized coordinates. A consequence of this is that the un-actuated generalized co-ordinates are indirectly controlled by means of actuated coordinates. This is done by means of dynamic coupling which is often non-linear. To add to the complexity, the wheels which drive the system are mounted on the shafts of the motor which are directly connected to the pendulum body.

It is difficult yet possible to apply the conventional control techniques to WIP since there are lesser number of actuators compared to the number of degrees of freedom to be stabilized. The dynamics of WIP are described by coupled non-linear differential equations [4]. It is however possible to approximate the dynamics around the operating point as a linear system, when the signals are small enough, such as for instance in a study conducted by Zhijun et al. [8], the model was linearized around the operating point and control was designed based on the linear model. Salerno et al. [9] studied the dynamics of WIP robot involving the pitch of the inverted pendulum. They considered the angular displacement of wheels (i.e rotation) as variables and designed a robust linear controller to stabilize the robot [9]. In this paper, the possibility of using neural networks for stabilizing WIP robot has been investigated.

\section{MODELING OF WIP SYSTEM}

We have considered a WIP system as shown in Figure. 1. It consists of an inverted pendulum, a pair of wheels and motor/actuators, motion controller and a chassis. The chassis supports the inverted pendulum as well as the wheels. The motion controller controls the wheels via actuators.

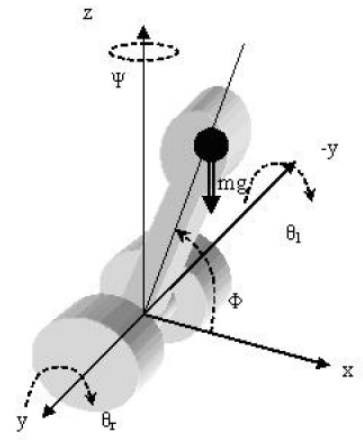

Fig. 1. WIP System concept 
In this set up, the system can be balanced on two wheels, it can also move forward and backward as well as spin about the vertical axis i.e. yaw. Table 1 identifies the various quantities and variables used in the model.

Although a simplified approach is adopted while developing the mathematical model, all the relevant dynamics of the system are captured. It is assumed that two onboard motors apply torque to each wheel as a reaction angular displacement produced in the pendulum. We are assuming that the wheels do turn but with respect to the pendulum body, which pitches under the reaction torque applied to the motors. It is also assumed that the wheels do not slip on the ground surface. The pendulum does not move along the $\mathrm{x}$-axis while it is being controlled in the upright position.

In this study, the torque is induced in the armatures of motors and applied on the wheels. Consequently, a reaction torque in opposite direction (clockwise direction) is applied on the chassis of the pendulum that is the vector sum of these two torques. The net torque along the $\mathrm{y}$-axis is given by (1).

$$
\tau_{y}=J \phi=\left(\tau_{r}+\tau_{l}\right)-m g l \cos \phi
$$

TABLE 1: NOMENCLATURE

\begin{tabular}{|c|c|}
\hline Symbol & Quantity \\
\hline$\varphi$ & Pitch Angle \\
\hline$\psi$ & Yaw Angle \\
\hline$\theta$ & Wheel Angle \\
\hline$\tau$ & Torque \\
\hline$m$ & Pendulum Mass \\
\hline$J$ & Net Rotational Inertia \\
\hline$r$ & Wheel Radius \\
\hline$d$ & Distance b/w wheels \\
\hline$l$ & Pendulum Length \\
\hline$g$ & Gravity Acceleration \\
\hline$J_{w}$ & Wheel Rot. Interia \\
\hline$L$ & Inductance \\
\hline$R$ & Resistance \\
\hline$b$ & Damping Coefficient \\
\hline$K_{t}$ & Motor Torque const. \\
\hline$K_{m}$ & Motor Back emf const. \\
\hline$i$ & Armature Current \\
\hline$v$ & Applied Voltage \\
\hline
\end{tabular}

Combining the reaction torques on motor together as $2 \tau_{\mathrm{m}}$ under the assumption that motors are connected in parallel, the resulting input torque on each motor is given by (2).

$$
J \ddot{\phi}+m g l \cos \phi=\tau_{m}
$$

Assuming that two DC motors with constant field current are used to drive wheels, the equations governing the induced torque in $i^{\text {th }}$ motor is given as:

$$
\begin{gathered}
v_{i}=R i_{a}+L \dot{i_{a}}+K_{m} \dot{\phi} \\
\tau_{i}=K_{t} i_{a}
\end{gathered}
$$

In this paper we are proposing WIP system model as a hybrid automaton. There are four discrete modes of the system namely pump energy, remove energy, wait and stabilize using neural controller. In the fourth discrete state, the neural controller comes into play to stabilize the WIP in upright position. The formal description of WIP as a hybrid automaton is given in Table 2. The model shows that when pendulum is lying flat either forward or backwards, the mode of system is energy pump (which corresponds to discrete state a) and torque is applied on the system so that the motion is directed towards the desired upright position.

When angular position comes within $[\pi / 2-\delta$, $\pi / 2+\delta]$, (here we have defined interval by setting $\delta$ equal to a value of $3 \pi / 20$, which was found experimentally), the remove energy mode of operation is activated (discrete state $b$ )

\section{TABLE 2: WIP AS HYBRID AUTOMATON}

$$
\begin{aligned}
& \begin{array}{l|l}
H=\{Q, X, \text { Init }, f, D, E, G, R\} & E(q)=\{(a, b),(b, c),(c, b),
\end{array} \\
& Q=\{a, b, c, d, e\} \quad(c, d),(a, c)\} \\
& X=\{\phi, T \in \Re\} \quad G(a, b)=\{\pi / 2-\delta \leq \phi \leq \pi / 2+\delta\} \\
& \begin{array}{l|l}
\text { Init }=Q \times(0,0)^{T} & G(b, a)=\{\phi>\pi / 2+|\delta|\}
\end{array} \\
& f(a, X)=\ddot{\phi}=\frac{\xi \tau-m g l e a \phi}{j} \quad G(b, c)=\{\phi=\pi / 2\} \\
& f(b, X)=\ddot{\phi}=\frac{-\xi r-m g l c o \phi}{J} \quad G(c, d)=\{\phi=0 \wedge T=0\} \\
& \begin{array}{l|l}
f(c, X)=\bar{\phi}=\frac{- \text { mgleo }_{\text {co }}}{J} & G(c, b)=\{\phi \neq \pi / 2\}
\end{array} \\
& f(d, X)=\{\text { determined by } N N\} \quad R(e)=\phi \forall e \in E \\
& D(a)=\{\Phi \in(\pi, \pi / 2+\delta) \\
& \vee(\pi / 2-\delta, \pi)\} \\
& D(b)=\{\phi \mid \Phi \in[\pi / 2+\delta, \pi / 2-\delta]\} \\
& D(c)=\{\phi, T \mid T<1\} \\
& D(d)=\{\phi=\pi / 2\}
\end{aligned}
$$


in which the applied torque is such that it opposes the motion away from $\pi / 2$, thus removing energy. As the system stops at the required upright position $\pi / 2$ (which corresponds to discrete state $c$ ), the system is forced to wait till the pendulum momentarily stops. If during this state the angle is not equal to $\pi / 2$, then system returns back to state b. Otherwise if it is, then state $d$ is initiated. In $d$, the neural controller stabilizes the system (ref to discrete state $d$ ). A variable $\xi$ is introduced so the sign of torque is such that the motion is always directed towards the upright position in $a$ and away in $b[19,20]$.

\section{SIMULATION MODEL OF WIP SYSTEM}

A simulation model was developed based on equations (1) to (4) as shown in Figure 2. The simulation model was developed using the Simulink software (MathWorks, US). The output signal of the system was pitch angle $\varphi$. Since the pendulum could either fall forward or backwards, "integrator" with saturation in range of $(0, \pi)$ radians was used. Furthermore, the initial conditions which refer to the position of the pendulum could be set in the same integrator. The model had two inputs, which were the two voltage inputs for the motors. It was observed that with initial condition equal to $\pi / 2 \mathrm{rad}, \varphi$ did not change. However, it would settle to $\pi \mathrm{rad}$ or $0 \mathrm{rad}$ if the initial condition was slightly above or below $\pi / 2 \mathrm{rad}$, respectively.

The model developed in the previous section was calibrated based on the data given in Table 3. Some of parameters were obtained from [10].

\section{NEURAL CONTROL OF WIP}

The field of neural networks is relatively new; the mathematical notations and architectural representations are therefore in a process of getting firmly established [11]. Furthermore,
TABLE 3 : WIP PARAMETERS

\begin{tabular}{|c|c|c|}
\hline Symbol & Value & Unit \\
\hline$m$ & 15 & $\mathrm{~kg}$ \\
\hline$J$ & 0.28 & $\mathrm{kgm}^{2}$ \\
\hline$r$ & 0.106 & $\mathrm{~m}$ \\
\hline$d$ & 2 & $\mathrm{~m}$ \\
\hline$l$ & 0.4 & $\mathrm{~m}$ \\
\hline$g$ & 9.8 & $\mathrm{~m} / \mathrm{sec}^{2}$ \\
\hline$J_{w}$ & 0.1 & $\mathrm{kgm}^{2}$ \\
\hline$L$ & 1 & $\mathrm{H}$ \\
\hline$R$ & 0.1 & $\Omega$ \\
\hline$b$ & 1 & $\mathrm{Ns} / \mathrm{m}$ \\
\hline$K_{t}$ & 100 & $\mathrm{Nm} / \mathrm{A}$ \\
\hline$K_{m}$ & 10 & $\mathrm{Vs} / \mathrm{rad}$ \\
\hline
\end{tabular}

the publications on neural networks are based on diverse disciplines, including but not limited to mathematics, psychology, engineering, physics, etc. Hence, most of the terminology used in these works is typical to the researcher's own field [12]. Neural Network and its application to controlling dynamic systems is itself a new approach of solving the control problem. The application of neural networks for controlling WIP system is an active research area, for instance C. Yang et al investigated the motion control of WIP using neural networks [3]. In their study, they investigated the motion control of WIP system model, where the WIP system was "decomposed" into a sub-system consisting of planar motion including yaw motion and a sub-system consisting of unactuated system modeling the pendulum's tilt motion. An adaptive neural network approach has been used for motion control of subsystem.

However, in this paper we have investigated the possibility of using a type of neural network called multiple layer perceptron (MLP) to control the Hybrid WIP system model. There are several other architectures in addition to MLP that can also be used [13]. The MLP network is pretty straightforward to be employed for the discrete-time modeling of 
the dynamic systems containing nonlinearities [14]. This means that MLP network can not only be used for system identification but also to control, when used as a controller. The MLP need not be in feed-forward structure, it may incorporate feedback and such MLP network is called recurrent network. There are many topologies of MLPsbased-control of dynamic systems such as neural network based stabilizing controller, adaptive inverse controller, nonlinear internal model control, model predictive control or neural adaptive control to name a few ( [15], [16]).

In order to implement the neural networks for control based on system identification, a neural network model structure needs to be identified as an initial step, out of several available choices that may be used for solving the problem at hand. There are several structures available based on MLP neural networks such as finite impulse response model structure (NNFIR), autoregressive external input (NNARX), autoregressive moving average external input (NNARMAX), state space innovation form (NNSSIF), output error (NNOE) to name a few. From stability point of view, NNFIR and NNARX model structures are preferred [17]. As a second part of this step, the structure of MLP is determined to find the number of neurons required to be present in the hidden layer and output layer so that the structure can adequately fulfil the modeling requirements. These structures can be used when plant model, inverse model of plant or NN model of controller is to be trained.

In this paper the MLP model developed by Yasir Amir et al. [18], containing training algorithm with necessary modifications has been used. We have investigated the use of the direct inverse neural control model structure with 50-100-1 MLP architecture (50 inputs, 100 hidden neurons, and one output neuron), with a sampling rate of 1000 samples/sec, since MLP of this complexity performed reasonably based on the simulation results. Based on our research, we state that, no imperial formula has been developed yet, to determine the exact size and complexity of network required to solve given control problem.

The simulation model thus developed using Simulink, was simulated using NN based controller implementing the direct inverse scheme [11,18]. Inverse model of WIP was developed and inserted so as to provide the target value of voltage output which is required to train the NN. As the simulation is started the "In Range?" subsystem signals the "switch A" to apply a dc voltage of $5 \mathrm{~V}$ (referred to as range parameter $=5$ ) to both the motors. The sign of voltage is such that the motion due to reaction torque on the WIP body is directed towards the upright position. As the pendulum moves and enters into the range (defined earlier), a reversed voltage with a magnitude of $1 \mathrm{~V}$ (referred to as reverse range parameter $=1$ ) is used to apply a counter torque to stop the pendulum. Thus, the system enters the discrete state $b$. When the pitch angle becomes close to $\pi / 2$, the discrete state $c$ is activated and finally after an interval of $T>1$, the neural controller is activated.

\section{SIMULATION RESULTS AND DISCUSSION}

The simulation of model reveals the results as shown in Figure 3. It should be noted that the sampling rate is set to 100 samples/sec. Initially, the pitch angle gradually increased until it reached the steady state after which it started oscillating vigorously. This is followed by the decrease in the amplitude of oscillations for a brief period of time, which again increases until the neural controller has been activated. The neural controller in this 
particular case was activated at $21.5 \mathrm{~s}$ with a learning rate of 0.01 (value chosen keeping in mind the simulation results). The neural controller then takes over the control of the plant. It can be observed that as the time passes the magnitude of oscillation decreases. After simulation for 50 s there is no significant decrease in the amplitude of oscillations. The error from the desired value obtained is $12.7 \%$. Figure 4 shows the result with range parameter changed to 4.9. It was observed that the error was related to the sampling rate. When the sampling rate was increased from 1000 to 10,000 samples/sec, the results obtained showed improvement as the steady state error decreased to less than 5\%. Figure 5 shows that discrete state $d$, was activated earlier than the previous case at $11.5 \mathrm{~s}$ and as shown, the steady state error reduced and steady state was achieved earlier.

The results show that as the sampling rate increases the steady state accuracy increases and settling time as well as time required to achieve the discrete state $d$ reduces. The experimental results depict a correlation between the sampling rate and steady state accuracy.

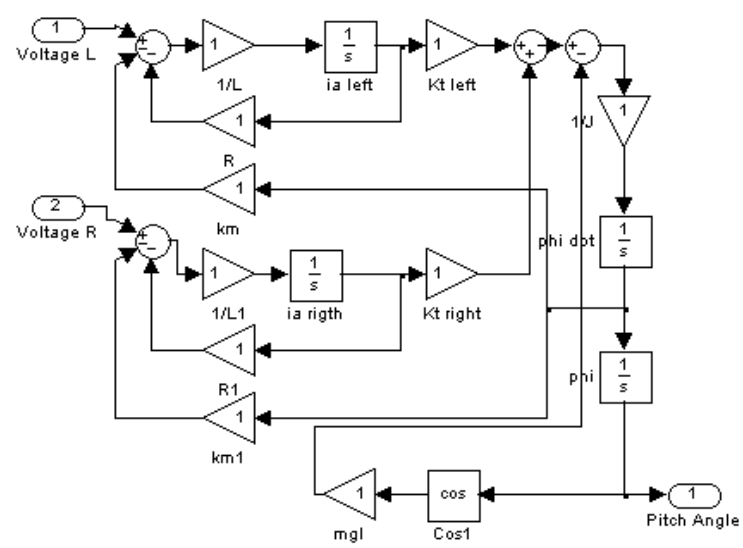

Fig. 2. Simulink ${ }^{\circ}$ Model of WIP System.

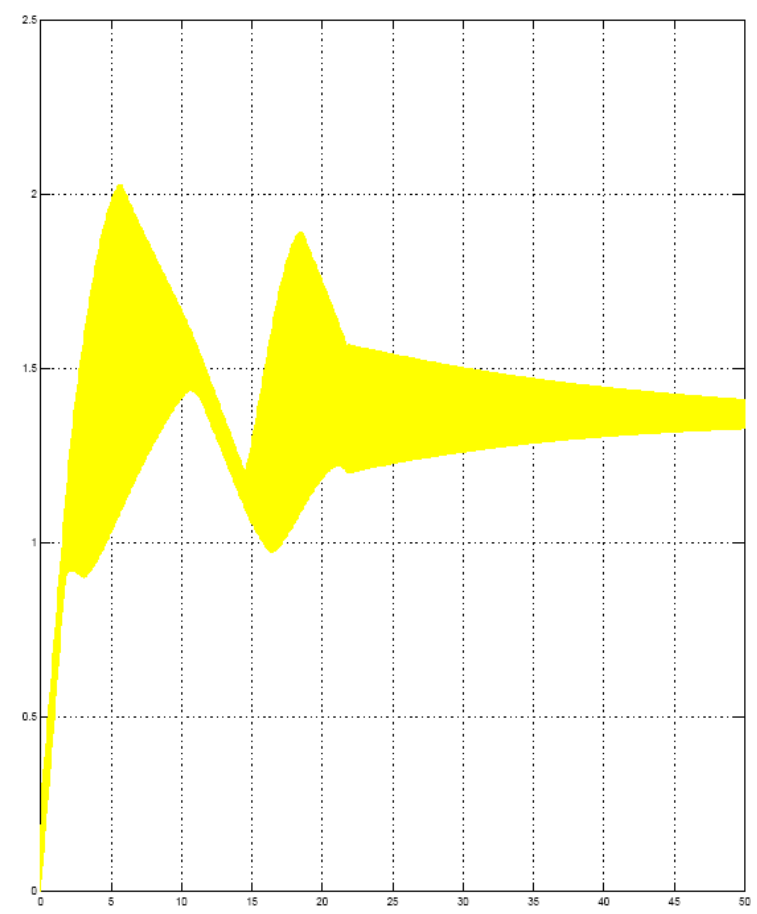

Fig. 3 Pitch Angle (in radians) versus time settles near $\pi / 2$ radian with error.

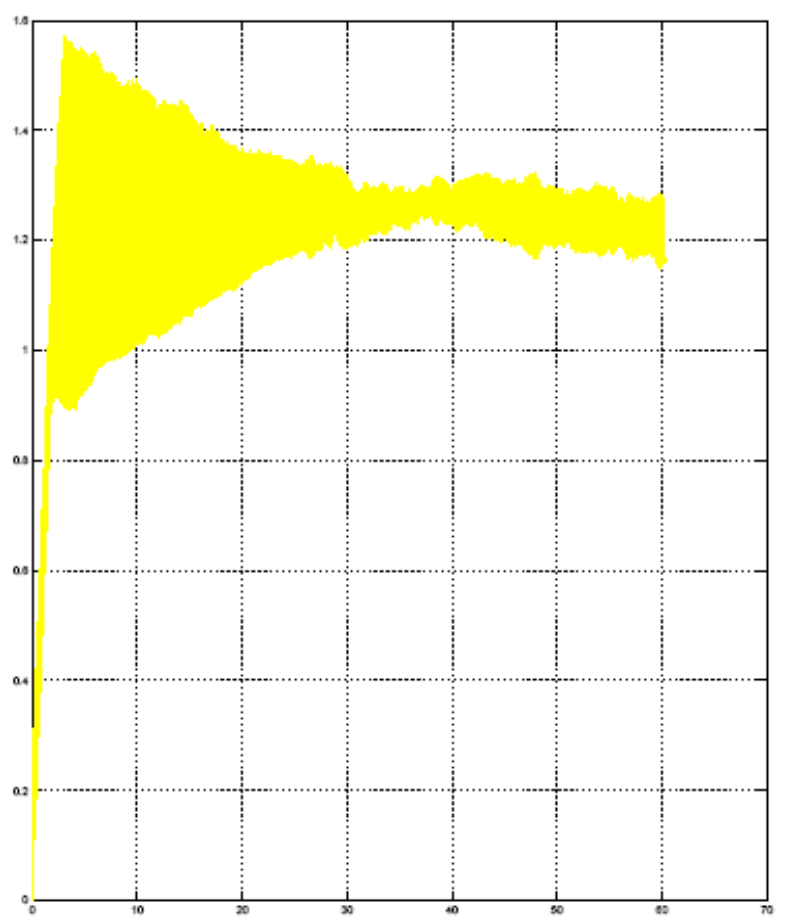

Fig. 4. Pitch Angle versus time with range parameter=4.9. 


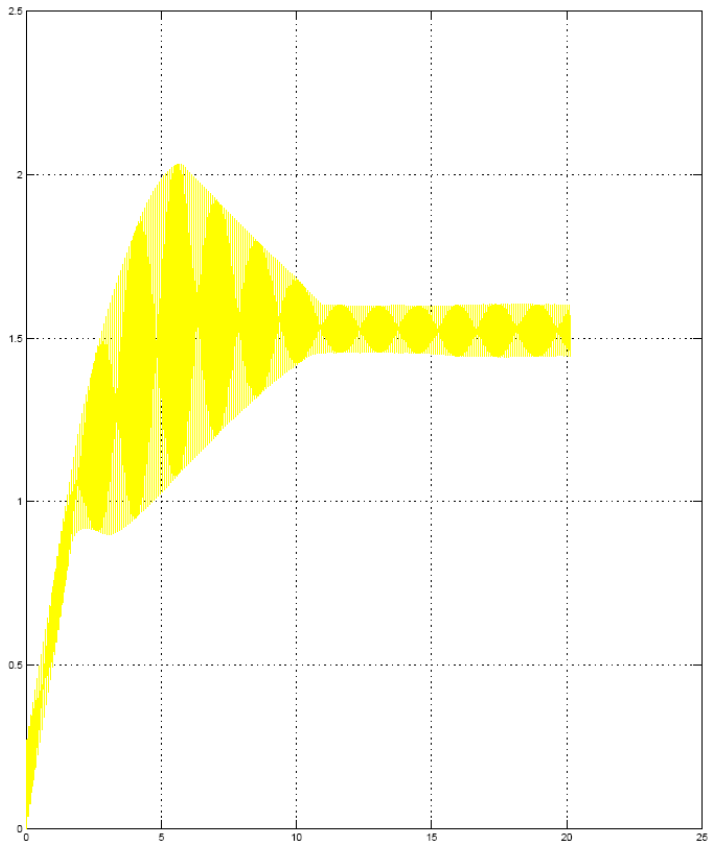

Fig. 5. Pitch Angle versus time with sampling rate increase to 1000 sample/sec.

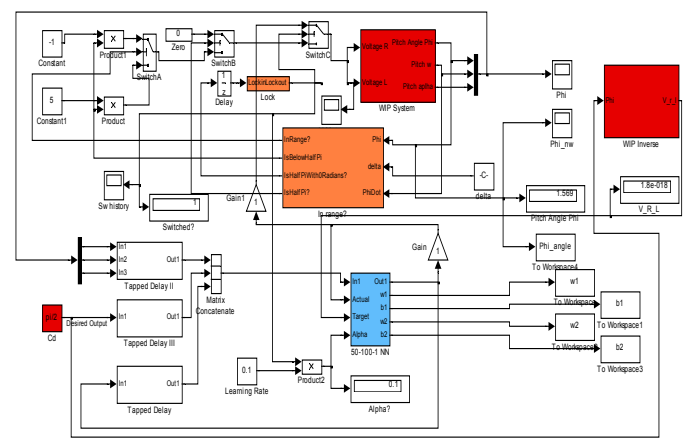

Fig. 6. Simulink ${ }^{\circledR}$ Model of the NN Controlled WIP System

\section{CONCLUSION}

The design and simulation of neural network based control of a WIP system modelled as a hybrid automaton was presented in this paper. Simulation results were also presented in this paper which revealed that this scheme successfully controlled the pendulum at desired position. It was found that the NN controller with 50 inputs, 100 hidden neurons, and 1 output neuron was able to keep the pendulum at the desired position. It was also observed that by increasing the sampling rate, the accuracy of output at desired position increased.

\section{REFERENCES}

[1] M. Askari, H. Mohamed, M. Moghavvemi, and S. S. Yang, "Model predictive control of an inverted pendulum," in Proc. of International Conference for Technical Postgraduates (TECHPOS), Kuala Lumpur, Malaysia, pp. 1-4, December 2009.

[2] K. Goher, S. Ahmad, and O. M. Tokhi, "A new configuration of two wheeled vehicles: Towards a more workspace and motion flexibility," in Proc. of IEEE International Systems Conference, pp. 524-528, 2010.

[3] C. Yang, Z. Li, R. Cui, "Neural Network-Based Motion Control of Underactuated Wheeled Inverted Pendulum Models," IEEE Trans. Neural Ntw. Learning Syst., vol. 25, pp. 2004-2016, 2014.

[4] Zhijun Li, Chenguang Yang, Liping Fan, "Introduction," in Advanced Control of Wheeled Inverted Pendulum System, London, UK: Springer, ch.1, sec. 1.1-1.3, pp 2-7, ISBN 978-14471-2962-2, 2013.

[5] David P Anderson. (2014, Nov 27).[Online]. Available: http://www.geology.smu.edu/ dpawww/robo/nbot/

[6] Victor Vicente Abreu, "Balance Bot," M.S. dissertation, Dept. Math and Eng., Madeira Univ., Funchal, Potugal, 2009.

[7] University of Illinois Urbana Champaign. (2014, Nov 27). Final Project of Introduction to Mechatronics. [Online]. Available: http://coecsl.ece.illinois.edu/ge423/spring04/grou p9/http://www.atm.com

[8] Grasser, F., Arrigo, A., Colombi, S., Rufer, "JOE: a mobile, inverted pendulum," IEEE Trans. Ind. Electron., vol. 49, pp. 107-114, 2002.

[9] Salerno, A., Angeles, J., "On the nonlinear controllability of a quasiholonomic mobile robot," in Proc. IEEE International Conf. Robotics and Automation, pp. 3379-3384, 2003.

[10] Nawawi, Ahmad, Osman,"Real-Time Control System for a Two- Wheeled Inverted Pendulum Mobile Robot," Advanced Knowledge Application in Practice, Shanghai, China: InTech, pp 299-313, ISBN: 978- 953-307-141-1, 2010.

[11] Yasir Amir Khan, "Artificial Neural Networks and Multiple Layer Perceptron", in Modeling and Neural Control of Quadrotor Helicopter, Berlin, Germany: LAP LAMBERT Academic Publishing AG \& Co, ISBN-13: 9783838392981, pp 24-33, 2011. 
[12] V. U. Abbas, Y. Amir, "Multiple layer perceptron for Direct Inverse Control of a nonlinear system," in Proc. IEEE International Conference on Computer Control and Communiction, Karachi, pp. 1-5, 2009.

[13] T. Koskela, M. Lehtokangas, J. Saarinen, K. Kaski, "Time Series Prediction with Multiple Layer Perceptron, FIR and Elman Neural Network," in Proc. of World Congress on Neural Networks, San Diego, CA, USA, pp 490, 1996.

[14] M. Norgaard, O. Ravan, N. K. Poulsen, L. K. Hansen, "Introduction: Introduction to Multilayer Perceptron Networks," in Neural Networks for Modeling and Control of Dynamic Systems, Berlin, Germany: Springer, ISBN 185233-227-1, pp 8-9, 2003.

[15] M. T. Hagan, H. B Demuth and M Beale, Neural Network design, PWS, 1996.

[16] Hagan, M.T. and Demuth, H.B., "Neural networks for control", in Proc. of American Control Conference, San Diego, CA, pp. 16421656, 1999.
[17] M. Norgaard, O. Ravan, N. K. Poulsen, L. K. Hansen, "System Identification with Neural Networks," in Neural Networks for Modeling and Control of Dynamic Systems, Berlin, Germany: Springer, ISBN 1-85233- 227-1, 2003, pp 16-30.

[18] Yasir Amir, V. U Abbas, "Modeling and Neural Control of Quad Rotor Helicopter," Yanbu J. Engineering and Science, vol.2, pp 35- 43, ISSN 1658-5321, 2011.

[19] Nancy Lynch, Bruce Krogh, Hybrid Systems: Computation and Control: Third International Workshop, HSCC 2000 Pittsburgh, PA, USA, March 23 - 25, 2000.

[20] Sebastian Engell, H. Guéguen, J. Zaytoon, Analysis and Design of Hybrid Systems 2003 (ADHS 03): A Proceedings Volume from the IFAC Conference, St. Malo, Brittany, France, Published by Elsevier, 16-18 June 2003. 
النمذجة، المحاكاة والتحم في بندول مقلوب العجلات بإستخدام الخلايا العصبية

محمد باسر أمير خان،، ولي الدين عباس2

1 قسم الهندسة الكهربائية، جامعة بهريا، الباكستان

2 قسم الهندسة الكهربائية، جامعة هدارد، الباكستان

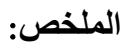

البندول مقلوب العجلات نظام غير مستقر للغاية. هذا البحث يقدم مقترح الندذجة والححاكاة والتحكم في بندول مقلوب العجلات كنموذج هجين معتمداً على أساس معادلات عزم الدوران بدلا من طريقة لاجر انج. تمت محاكاة النموذج باستخدام Simulink معتمداً على طريقة التحكم المباثر العصبي المعكوس باستخدام استر اتيجية الهجينة الالية وذللك للحفاظ على النظام في وضع مستقيم معكوس. وأوضحت نتائج الدحاكاة أن نظام التحكم المقترح قادر بدرجة كافية علي التحكم و الإبقاء علي النظام في الوضع المطلوب ولكن مع خطأ بسيط في الحالة المستقرة و هذا الخطأ وجد أنه للحد من زيادة

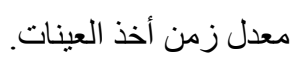

